\title{
Preparation of Colloidal Organosilica Spheres through Spontaneous Emulsification
}

\author{
Casper van der Wel, ${ }^{\dagger}$ Rohit K. Bhan, ${ }^{\ddagger}$ Ruben W. Verweij, ${ }^{\dagger}$ Hans C. Frijters, ${ }^{\dagger}$ Zhe Gong,
} Andrew D. Hollingsworth, ${ }^{\S}$ Stefano Sacanna, ${ }^{\ddagger}$ and Daniela J. Kraft*,†

${ }^{\dagger}$ Soft Matter Physics, Huygens-Kamerlingh Onnes Laboratory, Leiden University, P.O. Box 9504, 2300 RA Leiden, The Netherlands

${ }^{\ddagger}$ Molecular Design Institute, Department of Chemistry, New York University, 29 Washington Place, New York, New York 10003, United States

${ }^{\S}$ Center for Soft Matter Research, Department of Physics, New York University, 4 Washington Place, New York, New York 10003, United States

\section{Supporting Information}

ABSTRACT: Colloidal particles of controlled size are promising building blocks for the self-assembly of functional materials. Here, we systematically study a method to synthesize monodisperse, micrometer-sized spheres from 3-(trimethoxysilyl)propyl methacrylate (TPM) in a benchtop experiment. Their ease of preparation, smoothness, and physical properties provide distinct advantages over other widely employed materials such as silica, polystyrene, and poly(methyl methacrylate). We describe that the spontaneous emulsification of TPM droplets in water is caused by base-catalyzed hydrolysis, self-condensation, and the deprotona-

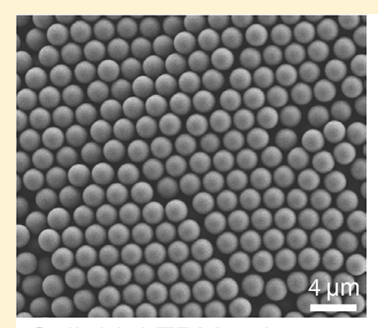

Colloidal TPM spheres

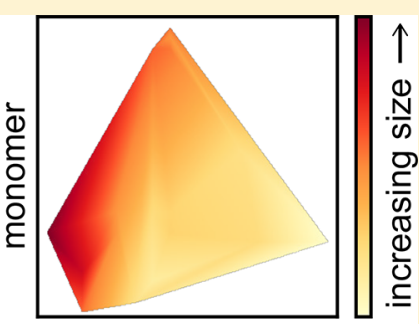

$\mathrm{pH}$ tion of TPM. By studying the time-dependent size evolution, we find that the droplet size increases without any detectable secondary nucleation. Resulting TPM droplets are polymerized to form solid particles. The particle diameter can be controlled in the range of 0.4 to $2.8 \mu \mathrm{m}$ by adjusting the volume fraction of added monomer and the $\mathrm{pH}$ of the solution. Droplets can be grown to diameters of up to $4 \mu \mathrm{m}$ by adding TPM monomer after the initial emulsification. Additionally, we characterize various physical parameters of the TPM particles, and we describe methods to incorporate several fluorescent dyes.

\section{INTRODUCTION}

Colloidal particles are widely used in industrial applications and also serve as model systems for fundamental studies, such as crystallization, ${ }^{1,2}$ the packing of small clusters, ${ }^{3}$ and the glass transition. ${ }^{4}$ Many of these applications rely on the availability of spherical particles that have an accurately controlled size: for instance, crystallization requires spheres with a polydispersity below 6\%. ${ }^{5}$ Monodisperse colloidal spheres can also be employed as basic building blocks for fabricating more complex anisotropic structures, such as lock-and-key particles, colloidal molecules, and Janus particles. ${ }^{6-9}$

Therefore, the synthesis of micrometer-sized monodisperse spheres has become an indispensable technique in colloidal science. Synthesis protocols for a select range of widely used materials are well established, in particular for silica, ${ }^{10}$ polystyrene, ${ }^{11}$ and poly(methyl methacrylate). ${ }^{12}$ Each of these materials has its own advantages: for example, silane chemistry allows for a wide range of possible surface coatings, polystyrene particles can be density matched with water in order to exclude gravitational effects, and poly(methyl methacrylate) particles can be refractive index and density matched in an organic solvent, enabling accurate three-dimensional confocal imaging.

Despite the well-documented and mostly straightforward synthesis methods, many scientists refrain from synthesizing these particles themselves due to the basic chemistry infrastructure that is required. Here, we study a benchtop preparation of monodisperse colloidal particles that can be implemented with as little as a stirred beaker and thus is readily accessible to a wide range of scientists. This synthesis method employs the alkoxylsilane 3-(trimethoxysilyl)propyl methacrylate (TPM, see Figure 1). The advantage of this material is threefold: First, the preparation can be performed without extensive chemical equipment and can be completed within $4 \mathrm{~h}$. Second, we show that tuning the available synthesis parameters allows control over the particle size from 0.45 to $1.70 \mu \mathrm{m}$. Third, the organosilica material of the resulting colloidal<smiles>C=C(C)C(=O)OCCC[Si](OC)(OC)OC</smiles>

Figure 1. Structural formula of 3-(trimethoxysilyl)propyl methacrylate (TPM).

Received: May 1, 2017

Revised: July 17, 2017

Published: July 21, 2017 
spheres has the chemical versatility of silica, while the material is stable in water, its density is lower than that of silica, and its refractive index is similar to that of poly(methyl methacrylate). This combination of features opens up new experimental possibilities, for instance, the manipulation and three-dimensional tracking of dense suspensions. ${ }^{13}$

The preparation of TPM spheres begins with the spontaneous formation of TPM emulsion droplets in water. This phenomenon has been described previously for different alkoxysilanes in water under alkaline conditions. ${ }^{14}$ Since TPM can be polymerized at its methacrylate moiety, these emulsion droplets can also be used to fabricate solid colloidal particles, which has been used in the past to image Pickering emulsions ${ }^{15,16}$ as well as to make complex and anisotropic particles. $^{6,8,13}$ However, the preparation method of TPM spheres has not been studied systematically to date. In order to provide a reference for the TPM microparticle preparation, we here first review previous studies on the emulsification of trialkoxysilanes. Then we summarize the preparation method and several ways of including fluorescent dyes, measurements of material properties, a time-resolved measurement of the synthesis, and a study of the effect of various synthesis parameters. Finally, we describe and characterize a seeded growth method for creating TPM droplets with sizes of up to $4 \mu \mathrm{m}$.

Review of Previous Work. Obey and Vincent ${ }^{14}$ first showed that a monodisperse emulsion can be formed spontaneously by mixing an alkoxysilane, dimethyldiethoxysilane, with water or ethanol mixtures under alkaline conditions. They proposed a mechanism that required the base-catalyzed nucleation and growth of polyalkoxysilane in water from an initial single-phase system. The emulsions formed were thought to be kinetically stabilized against aggregation by negative charges on the droplet interface.

The same method was adapted to create emulsions of TPM stabilized by various inorganic nanoparticles and tetramethylammonium hydroxide (TMAH) ions, so-called Pickering emulsions. ${ }^{15,17}$ Stable emulsion droplets of diameters ranging from 10 to $100 \mathrm{~nm}$ were observed in the presence of these nanoparticles. For Pickering emulsions stabilized by magnetite particles, ${ }^{15}$ the droplet size increased with increasing TPM volume fraction and decreased when more of the magnetite particles were added at a constant TPM volume fraction. The emulsion is also formed when the TPM is mixed with other monomers such as methyl methacrylate and styrene, indicating that only relatively small amounts of TPM are needed to stabilize the droplets. ${ }^{18}$

Later it was found that TPM emulsions can also be obtained in the absence of nanoparticles. ${ }^{6,16}$ These emulsions had diameters of approximately $1 \mu \mathrm{m}$, which is much larger than the Pickering emulsions. The initially polydisperse emulsions were formed after 1 day using a mixture of TPM, water, and various quaternary ammonium salts at $\mathrm{pH} 9$ to $10 .^{16}$ By adding prehydrolyzed TPM dropwise to an ammonia solution until the droplets reached their target size, Sacanna et al. ${ }^{6}$ succeeded in making these TPM-in-water emulsions monodisperse. The advantage of using TPM is that these droplets could then be polymerized using a radical initiator, which resulted in solid dimpled spheres if the polymerization was initiated from the water phase. Furthermore, the droplets could be fluorescently labeled by the addition of a rhodamine dye covalently linked to the alkoxysilane APS (3-aminopropyl triethoxysilane) after droplet formation. ${ }^{8}$
Because TPM will hydrolyze and subsequentlyself-condensate in water, ${ }^{19,20}$ hydrolysis and self-condensation reactions are believed to be the driving forces for the formation of a TPM emulsion. ${ }^{14,16}$ An overview of these reactions is given in Figure S1. The rates of these processes increase strongly with the $\mathrm{pH}$ of the solution: at neutral $\mathrm{pH}$, only 1 to $5 \%$ of TPM monomers have reacted after $12 \mathrm{~h},{ }^{21-23}$ while the reported first-order rate constant for hydrolysis at $\mathrm{pH} 9$ is $1.2 \mathrm{~h}^{-1}, 22$ which means that $90 \%$ of the TPM monomers have hydrolyzed after $2 \mathrm{~h}$. Condensation reactions eventually lead to TPM oligomers that are built up from octamers. ${ }^{19,21}$ Indeed, Sacanna et al. ${ }^{24}$ confirmed the release of methanol during TPM emulsion formation by ${ }^{1} \mathrm{H}$ NMR, which indicates that the TPM is at least partially hydrolyzed in the emulsification process. The same TPM oligomers were found regardless of the type of base added, indicating that the oligomers grow by the same mechanism. ${ }^{21}$ This implies that, while only ammonia has been employed so far, any base could in principle be used to form a monodisperse TPM emulsion.

In addition to hydrolysis and self-condensation, deprotonation is known to happen for silicates and alkyl-substituted silanols. $^{25,26}$ Although there is no study available on the deprotonation of TPM, there is evidence that at $\mathrm{pH} 9.2$ TPM oligomers become negatively charged. ${ }^{27}$ Sacanna et al. ${ }^{24}$ proposed a mechanism where the TPM will produce its own charged surfactant consisting of deprotonated TPM at the TPM-water interface, which could contribute to the stabilization of the emulsion droplets. By analyzing bulk TPM-water surface tensions, Kraft et al. ${ }^{28}$ found that emulsions are formed if the bulk surface tension is below $10 \mathrm{mN} \mathrm{m}^{-1}$. Below this surface tension, an increasingly larger droplet surface area is created with decreasing surface tension. Increasing the concentration of hydrolyzed TPM lowers the interfacial tension, pointing again toward the stabilizing properties of deprotonated TPM molecules.

In this study, we describe a preparation method of micrometer-sized TPM spheres as well as methods of including several fluorescent dyes. Then we characterize the material properties of TPM spheres. To further understand the spontaneous emulsification of TPM, we then study the time evolution of the TPM droplets during a synthesis as well as the effects of various synthesis parameters on the particle size distribution. Finally, we describe a seeded growth method to obtain particles with diameters of up to $4 \mu \mathrm{m}$.

\section{EXPERIMENTAL SECTION}

Materials. 3-(Trimethoxysilyl)propyl methacrylate (TPM, 98\%), ammonium hydroxide $\left(28 \% \mathrm{NH}_{3}\right.$ in water), tetramethylammonium hydroxide (TMAH, 25\% in water), azobis(isobutyronitrile) (AIBN, $\geq 98 \%$ ), potassium peroxodisulfate (KPS, $\geq 99 \%$ ), 2-hydroxy-2methylpropiophenone $(\geq 97 \%)$, rhodamine B isothiocyanate (RITC, mixed isomers), fluorescein isothiocyanate isomer I (FITC, $\geq 90 \%$ ), 3(aminopropyl)triethoxysilane (APS, $\geq 98 \%$ ), dimethyl sulfoxide (DMSO, $\geq 99.9 \%)$, 1,3,5,7-tetramethyl-8-phenyl-4,4-difluoroboradiazaindacene (BODIPY, $97 \%)$, perylene $(\geq 99.5 \%)$, pyridine $(\geq 99.9 \%)$, and 2-ethylhexyl 4-methoxycinnamate (98\%) were purchased from Sigma-Aldrich and used as received. Sodium hydroxide $(\mathrm{NaOH}$, 98.5\%) was obtained from Acros Organics. All solutions were prepared from deionized water with $18.2 \mathrm{M} \Omega \mathrm{cm}$ resistivity, using a Millipore Filtration System (Milli-Q Gradient A10).

Emulsification. To prepare TPM emulsions in water, we used ammonium hydroxide as a catalyst. Ammonia solution $(15 \mathrm{~mL}, 1-$ $1000 \mathrm{mM}$ ) was added to a $100 \mathrm{~mL}$ plastic (polypropylene) beaker with a PTFE-coated stir bar. The use of glassware was avoided as TPM reacts with the glass surface ${ }^{20}$ and leaves visible patches that cannot be 
cleaned easily (Figure S2). The $\mathrm{pH}$ of the ammonia solution was measured using a $\mathrm{HACH} \mathrm{HQ} 40 \mathrm{~d} \mathrm{pH}$ meter with a calibrated IntelliCAL PHC101 standard gel filled $\mathrm{pH}$ electrode. $\mathrm{pH}$ measurements were not performed after the addition of TPM because we suspect TPM reacts with the glass surface of the $\mathrm{pH}$ probe. Throughout, the beaker was covered with Parafilm to prevent $\mathrm{pH}$ change due to ammonia evaporation. While the solution was stirred at $350 \mathrm{rpm}$ (medium-sized vortex), an accurate volume of TPM monomer was injected with a plastic pipet. During the first $20 \mathrm{~min}$ of the reaction, the solution gradually becomes more turbid and the TPM droplets observed at the start of the reaction disappear. At this point, the stirring speed was lowered and stirring continued at 200 rpm until a total stirring time of $2.5 \mathrm{~h}$.

Radical Polymerization. After the spontaneous emulsification of TPM, the emulsion droplets can be polymerized in a radical polymerization reaction. During this process, the methacrylate moieties of the TPM polymerize, yielding a rigid particle that can be stored at room temperature for years.

To polymerize a TPM emulsion, we transferred the emulsion to a capped vial and added $10 \mathrm{mg}$ of the radical initiator AIBN per $15 \mathrm{~mL}$ of the emulsion. To initiate polymerization, the solution was heated to $80{ }^{\circ} \mathrm{C}$ for $2 \mathrm{~h}$. To prevent sedimentation, the vial was tumbled by hand at $30 \mathrm{~min}$ intervals. In the case in which droplets sedimented too quickly, leading to coalescence and a polydisperse dispersion, polymerization in an oil bath under continuous tumbling was employed.

Alternatively, $0.5 \mathrm{mM}$ potassium peroxodisulfate (KPS) can be used to initiate polymerization. Similar to AIBN, polymerization of the TPM droplets with KPS at $80{ }^{\circ} \mathrm{C}$ for $2 \mathrm{~h}$ yields spherical and solid colloids. Here we note that KPS can also yield dimpled spheres through shell buckling. ${ }^{29}$ If heating the sample during polymerization is undesirable, then a photoinitiator (0.1 vol \% 2-hydroxy-2methylpropiophenone) can be employed. After illumination with ultraviolet light (a Rayonet photochemical reactor operating at $350 \mathrm{~nm}$ wavelength) for $1.5 \mathrm{~h}$, rigid spheres are obtained. See Figure S3 for SEM pictures of colloidal particles prepared with these alternative initiators.

To corroborate the radical polymerization mechanism, we monitored the change in the FTIR spectrum in samples of emulsified TPM droplets and polymerized TPM spheres (Figure S4). We found that the carbon-carbon double-bond vibration decreased by $63 \%$ after polymerization. Therefore, we estimate the unreacted monomer fraction of methacrylate moieties at $37 \%$.

Fluorescent Dyes. To enable fluorescence microscopy of the TPM particles, several fluorophores are available for integration into the particles. All dyes were added after droplet formation. The dispersion was stirred for an additional $15 \mathrm{~min}$ to ensure a good distribution throughout the droplets. Then the emulsion was polymerized as described in the previous section. Fluorescence and SEM images of the resulting particles can be found in Figure S5.

Silane chemistry was used to covalently link the dyes to the organosilica network of TPM. ${ }^{30,31}$ For example, RITC (576 nm emission) and FITC ( $525 \mathrm{~nm}$ emission) can be covalently attached to the amino group in 3-(aminopropyl)triethoxysilane (APS) as follows: $10.2 \mathrm{mg}$ of RITC and $8.6 \mu \mathrm{L}$ of APS were dissolved in $10 \mathrm{~mL}$ of DMSO and stirred overnight. Typically, $10 \mu \mathrm{L}$ of this RITC-APS solution was added per $1 \mathrm{~mL}$ of TPM emulsion. The dye solution can be stored at room temperature under light exclusion for months without degradation.

FITC is linked to APS as follows: $3 \mathrm{mg}$ of FITC and $22.5 \mu \mathrm{L}$ of APS were dissolved in $250 \mu \mathrm{L}$ of ethanol and stirred overnight. Typically, $2.3 \mu \mathrm{L}$ of this FITC-APS solution was added per $1 \mathrm{~mL}$ of TPM emulsion. For long-term storage, water must be excluded to prevent hydrolysis and self-condensation of the APS molecules. ${ }^{30}$

Because of the hydrophobic nature of TPM, it is possible to also include hydrophobic dyes: both BODIPY (508 nm emission, also available in other colors) and perylene (447 nm emission) were dissolved at $1 \mathrm{~g} / \mathrm{L}$ in DMSO. Typically, $10 \mu \mathrm{L}$ of one of these dye solutions is added per $1 \mathrm{~mL}$ of TPM emulsion in order to obtain fluorescent particles. These noncovalently incorporated dyes migrate from the particles when they are transferred to a hydrophobic solvent.

Imaging Methods. TPM emulsions were imaged using a Nikon Eclipse Ti microscope with a $100 \times$ oil-immersion objective (NA 1.4). Dried samples of polymerized particles were sputter coated with $\mathrm{Pt} / \mathrm{Pd}$ and imaged using a FEI nanoSEM 200 scanning electron microscope (SEM) using an acceleration voltage of $15 \mathrm{kV}$. The particles obtained through the seeded growth process were imaged on a Zeiss Merlin field emission SEM using an acceleration voltage of $1 \mathrm{kV}$. The SEM images were analyzed using ImageJ software and a Python algorithm ${ }^{32}$ to determine the particle size distribution.

\section{RESULTS AND DISCUSSION}

Physical Properties. The supplier-reported density of TPM monomer is $1.045 \mathrm{~g} \mathrm{~cm}^{-3}$. We measured the density of TPM in dispersion with an Anton-Paar DMA 4500 M density meter. From the total dispersion density at four different particle mass fractions, the density of self-emulsified TPM was determined to be $1.235(10) \mathrm{g} \mathrm{cm}^{-3}$. After polymerization, the density increased by approximately $7 \%$ to $1.314(4) \mathrm{g} \mathrm{cm}^{-3}$. Polymerized particles that were calcined for $2 \mathrm{~h}$ at $700{ }^{\circ} \mathrm{C}$ further increased in density to $2.094(16) \mathrm{g} \mathrm{cm}^{-3}$. All density measurements were performed at $20.0{ }^{\circ} \mathrm{C}$.

The refractive index of polymerized TPM particles was measured by a refractive index matching process. TPM particles were dispersed in a mixture of pyridine $\left(n_{\mathrm{D}}=1.509\right)$ and 2ethylhexyl 4-methoxycinnamate $\left(n_{\mathrm{D}}=1.545\right)$, and the scattering intensity of the dispersion was measured at angles of 60,120 , and $150^{\circ}$ with a static light scattering instrument (Scitech Instruments ST-100). The refractive index of TPM particles was estimated to be between 1.512 and 1.513, assuming that the fluids are nonsolvents for the particles. Refractive index measurements were performed with a standard Abbe refractometer (Edmund Optics) at room temperature $\left(22.5{ }^{\circ} \mathrm{C}\right)$.

The dielectric constant of liquid TPM was measured using a calibrated parallel plate capacitor and an HP4192A impedance analyzer (Hewlett-Packard) in the frequency range of 10 to 100 $\mathrm{kHz}$. The dielectric constant of liquid TPM was found to be $6.58(4)$ at $22.3{ }^{\circ} \mathrm{C}$.

The zeta potential of the polymerized TPM particles was measured using a Zetasizer Nano ZS (Malvern Instruments) on dilute aqueous samples $(\mathrm{pH} 5.6)$ at $25.0{ }^{\circ} \mathrm{C}$. We determined that the TPM particles were negatively charged for all synthesis parameters, with a zeta potential of $-36(3) \mathrm{mV}$.

Emulsification. In order to gain insight into the spontaneous emulsification of TPM in water, we studied the evolution of droplet size during synthesis. At given intervals following the TPM monomer injection, we sampled $100 \mu \mathrm{L}$ of the reacting dispersion and imaged a $10 \mu \mathrm{L}$ aliquot with light microscopy. In order to freeze the emulsification process so that the size distribution could be determined accurately, we polymerized each sample directly as follows: $1 \mathrm{~min}$ before sampling, a vial with $1 \mathrm{mg}$ of AIBN and $1 \mathrm{~mL}$ of water at $80^{\circ} \mathrm{C}$ was prepared. Then, a $90 \mu \mathrm{L}$ aliquot of the sample was added to the vial and polymerized for at least $2 \mathrm{~h}$ in an $80{ }^{\circ} \mathrm{C}$ oven.

With SEM, we observed that the particles remained spherical during polymerization (Figures 2 and S4). Next to these TPM spheres, sub-200-nm objects were visible that decreased in number throughout the process. As we did not detect these before polymerization, we concluded that they are an artifact of the employed SEM sample preparation. Possibly, the incompletely reacted TPM species polymerize from solution. As these objects tended to precipitate on the growing droplets, 

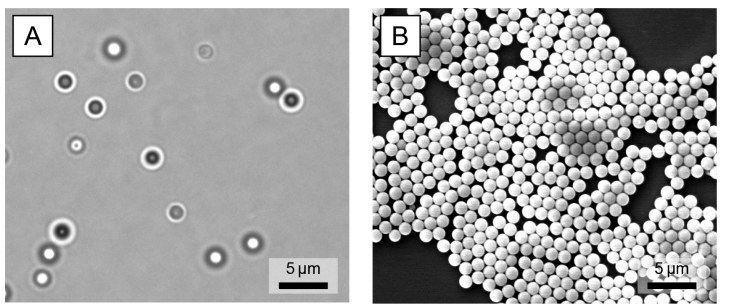

Figure 2. Microscopy images of TPM droplets. (A) Optical microscopy image of unpolymerized TPM droplets. (B) SEM micrograph of the same dispersion after polymerization.

the smoothness of the particles could be confirmed only at the end of the process. For the size measurements, we therefore washed the polymerized samples two times with water. Note that we measure the diameter of the polymerized TPM particles: as the density of polymerized TPM is 7\% higher with respect to the emulsion droplets, we expect the particles to be smaller accordingly.

The temporal evolution of the size distribution during a synthesis in ammonia solution at initial $\mathrm{pH} 10.4$ and TPM volume fraction $\phi=0.005$ is shown in Figure 3. Corresponding SEM images can be found in Figure S6. We define $t=0$ at the time of TPM monomer injection. At $t=10 \mathrm{~min}$, ca. $200 \mathrm{~nm}$ TPM droplets were observed by light microscopy. At $t=15$ min, spherical particles with a unimodal diameter distribution of $0.47 \pm 0.05 \mu \mathrm{m}$ were identified using SEM. After this initially fast nucleation step, droplets grow with a decreasing growth rate (Figure 3B). As can be seen in Figure 3C, the polydispersity index decreases before plateauing to $2-4 \%$ at $t$ $=90 \mathrm{~min}$. After that period, the size distribution broadens slightly until the droplets reach a stable size of $1.49 \pm 0.07 \mu \mathrm{m}$.

On the basis of these observations, we propose the following mechanism for the spontaneous emulsification of TPM droplets (Figure 4). Although our mechanism does not capture the details of the nucleation process, it provides a qualitative
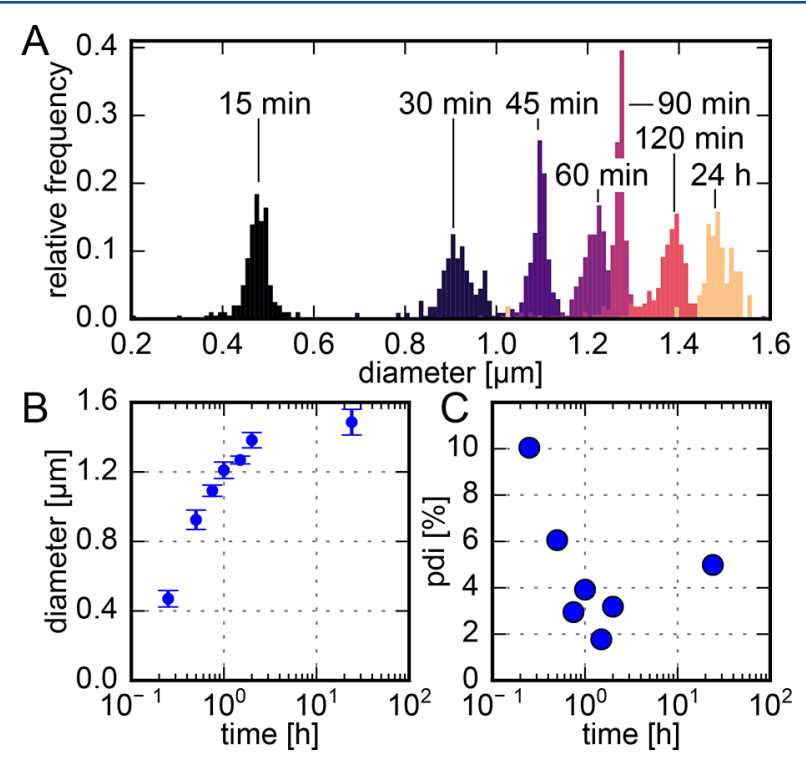

Figure 3. Temporal evolution of the size distribution of polymerized TPM droplets in a typical synthesis. (A) Size distributions at different time points in the synthesis. (B) The distributions show a strongly decreasing growth speed. (C) The polydispersity index (PDI) decreases initially and then slowly increases. description of the particle synthesis. Directly after monomer injection, TPM starts to hydrolyze and self-condensate (Figure S1) into oligomers of several monomer units due to a wellestablished cascade of base-catalyzed reactions. ${ }^{19,21,22}$ The gradual shrinkage of bulk monomer droplets has also been observed directly in the preparation of magnetite-covered TPM droplets. ${ }^{17}$ Because the oligomers become increasingly less soluble in water, they may self-nucleate or aggregatively nucleate (or both) to form the initial emulsion droplets. The accumulation of surface charge on the droplets due to silanol deprotonation prevents or limits the agglomeration of the small particles from forming larger ones. ${ }^{14,25,26}$ The influence of deprotonation will be further investigated in the next section by varying the $\mathrm{pH}$.

At this point, the number of droplets does not appear to change appreciably: we hypothesize that any insoluble oligomeric species with insufficient charge is taken up by the droplets. The dispersion gradually becomes turbid, and we observed the formation of an emulsion with an average droplet diameter of approximately $0.5 \mu \mathrm{m}$. Particles grow with decreasing growth rate by taking up TPM species in hydrolyzed, condensated, and/or coagulated form. The macroscopic monomer reservoirs are ultimately consumed during the growth of the droplets. Droplet growth is faster for smaller particles so that the size distribution of emulsion droplets will narrow with time. At a later stage, the polydispersity increases slightly, possibly due to competing processes such as Ostwald ripening.

Changes of $\mathrm{pH}$ and Monomer Fraction. The synthesis of TPM particles, as described in the preceding section, has only a few parameters, of which the most important ones are the $\mathrm{pH}$ and TPM monomer fraction. We demonstrate their effect on the resulting particle size and polydispersity by a systematic variation and polymerization after $2.5 \mathrm{~h}$ of emulsification. See Figure 5. Monodisperse emulsions formed only when the $\mathrm{pH}$ exceeded 9.7; below that value, highly polydisperse emulsions with sizes ranging from 2 to $20 \mu \mathrm{m}$ after $24 \mathrm{~h}$ of stirring were observed (Figure S7). Apparently, the hydrolysis and selfcondensation reactions proceed too slowly below $\mathrm{pH}$ 9.7. Also, colloidal stability is compromised under this condition due to insufficient deprotonation of the TPM species. Above $\mathrm{pH} 12$, we observed the formation of emulsions with a large spread of diameters ranging from 0.2 to $0.5 \mu \mathrm{m}$, which limits the synthesis conditions at the other end of the $\mathrm{pH}$ range.

For reaction conditions with initial $\mathrm{pH}$ values between 10.2 and 11.8 , we found that the particle diameter decreased monotonically for increasing $\mathrm{pH}$ (Figure 5B). The observed trend is supported by our proposed particle-formation mechanism: at higher $\mathrm{pH}$, TPM oligomerization occurs more rapidly. ${ }^{21,22,27}$ Silanol deprotonation is enhanced under these conditions as well, ${ }^{26}$ resulting in a larger number of primary droplets. Therefore, if the TPM volume fraction is kept constant, then smaller particles are produced as we observed. A shorter nucleation period is further supported by the observed narrowing of the size distribution with increasing $\mathrm{pH}$.

Similarly to previous experiments on magnetite-covered TPM emulsions, ${ }^{17}$ we found that the particle diameter increased with increasing monomer volume fraction. For a fixed $\mathrm{pH}$ of 10.8 (Figure 5C), the diameter of the emulsion droplets could be continuously tuned from 0.1 to $2.0 \mu \mathrm{m}$ by simply adjusting the TPM volume fraction from 0.05 to 5 vol \%. Below a volume fraction of 0.1 vol \%, the relative polydispersity increased to $20 \%$. This effectively sets a lower 


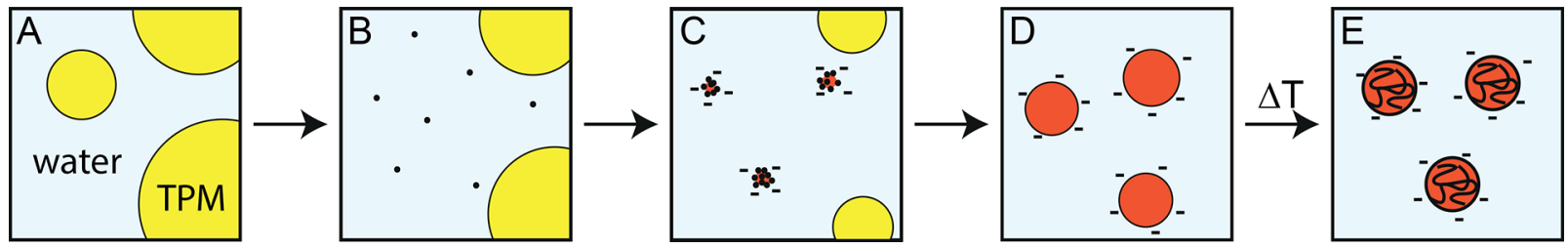

Figure 4. Schematic representation of the spontaneous emulsification and subsequent polymerization of TPM. (A) The process starts with TPM monomer injection with a two-phase system consisting of liquid TPM monomer (yellow) and water. (B) Hydrolysis and self-condensation of TPM result in oligomers of several monomer units (black dots). (C) Oligomers nucleate emulsion droplets (red) that are eventually stabilized by charge. The dispersion becomes turbid. (D) The droplets grow by acquiring more TPM species. In this stage, additional TPM monomer or dye solutions can be added that also transfer to the emulsion droplets. (E) In a second synthesis step, the methacrylate moiety of the TPM is polymerized by adding a radical initiator at elevated temperature.

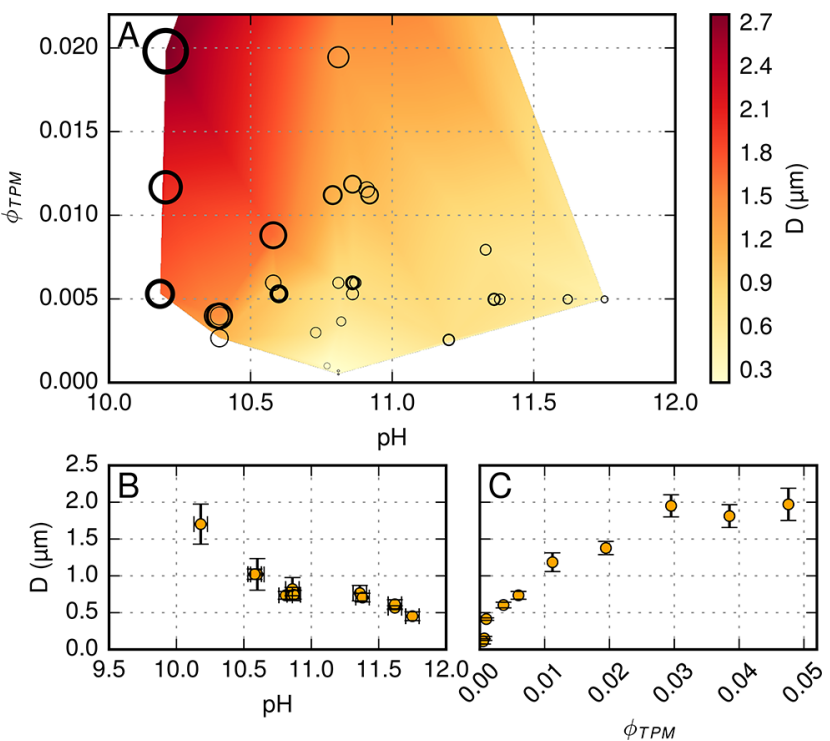

Figure 5. Plots of the influence of $\mathrm{pH}$ and TPM volume fraction on the diameter of TPM particles. (A) The diameter and line thickness of the plotted circles represent the diameter and polydispersity of the polymerized particles, respectively. The colors are linear interpolations between the measured points. (B) Particle diameter $D$ as function of $\mathrm{pH}$ at a fixed TPM monomer fraction of 0.005 to 0.006 . The particle size decreases as the $\mathrm{pH}$ increases. (C) Particle diameter as function of TPM monomer fraction $\phi$ at a fixed initial $\mathrm{pH}$ of $10.80(5)$. The particle size increases as the TPM monomer fraction increases.

limit of the TPM monomer fraction at 0.1 vol \%, which corresponds to a droplet diameter of $0.4 \mu \mathrm{m}$. Above a monomer volume fraction of $3 \mathrm{vol} \%$, the droplets did not grow significantly while the droplet polydispersity increased. Therefore, we conclude that at $\mathrm{pH} 10.8$ the practical range for the TPM volume fraction is 0.1 to $3 \mathrm{vol} \%$.

TPM Monomer Storage. To investigate the importance of the TPM monomer storage conditions, we also performed the synthesis using a TPM stock that was exposed periodically to air during a period of roughly 6 months. It is $\mathrm{known}^{21}$ that TPM slowly forms oligomers when contacted with small amounts of moisture such as is present in the air. Using the airexposed TPM, we synthesized particles and measured their average size as a function of monomer volume fraction and $\mathrm{pH}$ (Figure S8). We found the particle size to depend nonlinearly on the $\mathrm{pH}$. Above $\mathrm{pH}$ 9.7, a stable emulsion was formed, comparable to the synthesis with fresh TPM. Similarly, from $\mathrm{pH} 9.7$ to 10.7 , the particle size decreases monotonically. However, from $\mathrm{pH} 10.7$ to 12 , the particle size was found to increase again to a diameter of $1.47 \pm 0.14 \mu \mathrm{m}(\mathrm{pH} 12.0, \phi=$
$0.005)$, which contrasts sharply with the monotonous decrease to $0.45 \pm 0.06 \mu \mathrm{m}(\mathrm{pH} \mathrm{11.8,} \phi=0.005)$ observed for fresh TPM. The particle size polydispersity did not increase significantly due to the uncontrolled moisture exposure. These results indicate that the initial droplet formation, but not the droplet growth, is affected by the aging of TPM. Therefore, to ensure batch-to-batch reproducibility, we recommend handling the TPM monomer as a moisturesensitive compound, taking care to exclude or minimize exposure to air, for example by storing under a nitrogen atmosphere.

Influence of Base Species. To evaluate other base catalysts, we performed the particle synthesis using three different basic compounds: ammonia, sodium hydroxide $(\mathrm{NaOH})$, and tetramethylammonium hydroxide (TMAH). If the emulsification process were dependent on the basecatalyzed processes alone, then the type of base used to adjust the $\mathrm{pH}$ should not be important. ${ }^{21,26}$ Indeed, we observed that for all three bases a monodisperse emulsion was formed under appropriate conditions, confirming that $\mathrm{pH}$ is the control parameter for stable emulsion formation.

The results for the particle size as a function of $\mathrm{pH}$ and TPM volume fraction are presented in Figure S9 (TMAH) and Figure $\mathrm{S} 10(\mathrm{NaOH})$. For fixed monomer volume fraction $(\phi=$ $0.005)$, the resulting particles were $0.61 \pm 0.06 \mu \mathrm{m}$ when using ammonia at $\mathrm{pH} 11.6,0.72 \pm 0.03 \mu \mathrm{m}$ when using TMAH at $\mathrm{pH}$ 11.7, and $0.78 \pm 0.08 \mu \mathrm{m}$ when using $\mathrm{NaOH}$ at $\mathrm{pH} 11.8$. Because of the differences in base dissociation constants, the respective concentrations of these solutions were $0.9 \mathrm{M}$ ammonia $\left(\mathrm{p} K_{\mathrm{b}}=4.75\right), 0.4 \mathrm{M}$ TMAH $\left(\mathrm{p} K_{\mathrm{b}}=4.2\right)$, and 6.3 $\mathrm{mM} \mathrm{NaOH}\left(\mathrm{p} K_{\mathrm{b}}=0.20\right)$. Although there is a large difference in base concentration, the resulting particle sizes are very similar. This observation supports our claim that the synthesis process and resulting particle size depend mainly on $\mathrm{pH}$ and less on the type of base employed.

The fact that TMAH can be used to form monodisperse emulsions of TPM in water also sheds new light on previous experiments performed with TPM droplets that were stabilized by various inorganic colloids in the presence of TMAH. ${ }^{15,17,18,24,28,33}$ It was proposed that the formed TPM emulsions were stabilized by inorganic colloids at the interface, deprotonated TPM molecules, and TMAH that functions as a peptizing agent. Our study shows that the TMAH-induced deprotonation of TPM might be more important than the stabilization by TMAH itself. This conclusion suggests that TPM droplet formation is governed primarily by the volume fraction of TPM monomer and the $\mathrm{pH}$ of the solution only. 


\section{SEEDED GROWTH}

TPM droplets can be grown to larger sizes through a seeded growth process. After the initial emulsification of the droplets, additional TPM monomer is added to the stirred system to increase the diameter of the particles. A period of $1 \mathrm{~h}$ is necessary for complete hydrolysis and condensation of the first addition of oil-monomer, resulting in visibly larger emulsions. This process can be repeated multiple times, allowing growth to a desired particle size.

In a typical procedure, we add $500 \mu \mathrm{L}$ of TPM monomer $(\geq 98 \%)$ to $100 \mathrm{~mL}$ of ammonia solution at $\mathrm{pH} 11(1 \mathrm{~mL}$ of $2.8 \%$ ammonia and $99 \mathrm{~mL}$ of water in a stirred and covered glass beaker). Every hour, we added an additional $1 \mathrm{~mL}$ of TPM monomer to the emulsion. When using this technique to grow large droplets (above $2.5 \mu \mathrm{m}$ ), both the ratio of water-tooil and interparticle spacing decrease. At low water-to-oil ratios, a phase separation can occur, driving emulsion droplets back into the oil phase. This situation can be avoided easily by increasing the volume of the solution. Adding $100 \mathrm{~mL}$ of ammonia solution every fifth addition of monomer sustained particle growth. Larger particles can be grown in fewer intervals by increasing the amount of monomer added. We recommend adding no more than four times the original amount of monomer $(2 \mathrm{~mL}$ in our procedure). The addition of too much monomer can cause a phase separation between water and TPM monomer and thus a loss of particles.

This process results in very low particle polydispersity, as can be seen from the crystal lattice formation in the SEM images (Figures 6A and S11). The resulting particle diameters are shown in Figure 6B,C. Clearly, the particle volume increases linearly with the added monomer volume, indicating a pure growth process. This behavior is unlike the behavior for the initial emulsification step. The absence of secondary nucleation further corroborates the conclusion that the added TPM is distributed evenly over the TPM droplets.
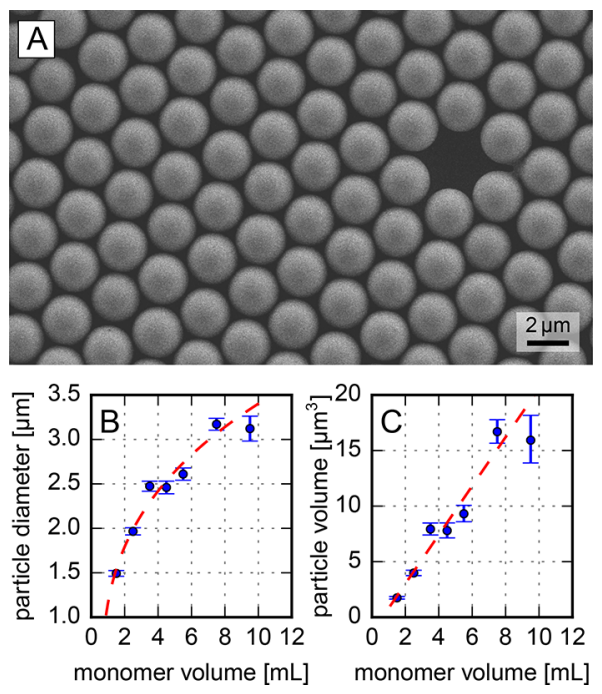

Figure 6. Dependence of TPM particle size on added monomer in a seeded growth process. (A) SEM micrograph of TPM particles that were obtained after the addition of a total of $4.5 \mathrm{~mL}$ of monomer. (B) Particle diameter as a function of total added monomer volume. (C) Particle volume as a function of total added monomer volume. The dashed red line in $(C)$ is obtained with linear regression. The same line is also displayed in (B). Corresponding SEM images can be found in Figure S11.

\section{SUMMARY AND CONCLUSIONS}

We have systematically studied a straightforward particle synthesis to prepare monodisperse colloids based on the spontaneous emulsification of a polymerizable trialkoxysilane. Also, we characterized the density, refractive index, dielectric constant, and zeta potential of the particles as well as several ways to include dyes for use in fluorescence microscopy. We found that the TPM particle size can be controlled via the $\mathrm{pH}$ and the volume fraction of added monomer to produce particles of 0.4 to $2.8 \mu \mathrm{m}$ in diameter. The particle size was observed to increase with increasing monomer volume fraction while the particle size decreased with increasing $\mathrm{pH}$. Furthermore, we showed that these trends hold regardless of which base is used as a catalyst.

We proposed a mechanism for the spontaneous emulsification of TPM droplets. First, the hydrolysis and selfcondensation of TPM produce oligomers that subsequently nucleate ca. $200 \mathrm{~nm}$ primary droplets. Deprotonation of the TPM species provides charge to these droplets. When the droplet charge is sufficiently large to ensure colloidal stability, the process enters a growth step in which the particle size increases without secondary nucleation. The relative polydispersity decreases in this growth step, although at longer times the particle size distribution broadened slightly.

For increasing synthesis $\mathrm{pH}$, we obtain smaller droplets. As a higher $\mathrm{pH}$ induces more charges in the droplet surface, deprotonation suppresses TPM oligomer coagulation at an earlier stage by means of electrostatic repulsion between droplets so that a (colloidally) stable emulsion with more droplets is obtained. Because the available TPM monomer is distributed over the droplets, a smaller size is obtained.

After the initial emulsification step, particles can be grown to sizes of up to $4 \mu \mathrm{m}$ by repeated addition of monomeric TPM. In this way, particles with a narrow size distribution can be obtained over a large size range from 0.4 to $4 \mu \mathrm{m}$.

TPM particles have already been proven to be a versatile template for the bulk synthesis of more complex particles. ${ }^{2,6,8,13}$ The organosilica nature of the TPM particles provides handles for many chemical functionalizations, and the fact that the size of the particles can be tuned by changing easily accessible parameters such as the $\mathrm{pH}$ or the amount of added monomer provides flexibility. In this method, no extensive chemical equipment is required: if, for instance, a dilute sodium hydroxide stock is used, then the synthesis can be performed as a tabletop experiment. The simplicity of this colloidal synthesis makes it easily accessible to a wide range of applications.

\section{ASSOCIATED CONTENT}

\section{S Supporting Information}

The Supporting Information is available free of charge on the ACS Publications website at DOI: 10.1021/acs.langmuir.7b01398.

Hydrolysis, deprotonation, and self-condensation reactions of TPM. Cover slides affected by the the reaction mixture. Syntheses with two alternative radical initiators. Infrared spectra of unpolymerized and polymerized droplets. Fluorescent particles. SEM micrographs showing the time evolution in a single synthesis. Results of syntheses at low $\mathrm{pH}$. Influence of $\mathrm{pH}$ and TPM volume fraction on the particle diameter in the case air-exposed TPM is utilized. Influence of $\mathrm{pH}$ and TPM volume 
fraction on the particle diameter when using TMAH or $\mathrm{NaOH}$ as base catalyst. SEM micrographs of particles obtained in a seeded growth process. (PDF) Bright field microscopy images during a TPM synthesis (AVI)

\section{AUTHOR INFORMATION}

\section{Corresponding Author}

*E-mail: kraft@physics.leidenuniv.nl.

\section{ORCID $\odot$}

Casper van der Wel: 0000-0002-0488-2237

Daniela J. Kraft: 0000-0002-2221-6473

\section{Notes}

The authors declare no competing financial interest.

\section{ACKNOWLEDGMENTS}

The authors thank Rachel Doherty for SEM imaging and Rob Kortschot for performing the dielectric constant measurements. This work was supported by The Netherlands Organisation for Scientific Research (NWO/OCW), as part of the Frontiers of Nanoscience program and through VENI grant 680-47-431. Additional financial support was provided by NASA (NNX13AR67G) and by the DURF award from New York University. The Zeiss SEM was purchased with financial support from the MRI program of the National Science Foundation under award DMR-0923251.

\section{REFERENCES}

(1) van Blaaderen, A.; Ruel, R; Wiltzius, P. Template-directed colloidal crystallization. Nature 1997, 385, 321-324.

(2) Wang, Y.; Wang, Y.; Zheng, X.; Ducrot, É.; Yodh, J. S.; Weck, M.; Pine, D. J. Crystallization of DNA-coated colloids. Nat. Commun. 2015, 6, 7253.

(3) Manoharan, V. N.; Elsesser, M. T.; Pine, D. J. Dense packing and symmetry in small clusters of microspheres. Science 2003, 301, 483487.

(4) Weeks, E. R.; Crocker, J. C.; Levitt, A. C.; Schofield, A.; Weitz, D. A. Three-dimensional direct imaging of structural relaxation near the colloidal glass transition. Science 2000, 287, 627-631.

(5) Pusey, P. N. The effect of polydispersity on the crystallization of hard spherical colloids. J. Phys. (Paris) 1987, 48, 709-712.

(6) Sacanna, S.; Irvine, W. T. M.; Chaikin, P. M.; Pine, D. J. Lock and key colloids. Nature 2010, 464, 575-578.

(7) Chen, Q.; Bae, S. C.; Granick, S. Directed self-assembly of a colloidal kagome lattice. Nature 2011, 469, 381-384.

(8) Sacanna, S.; Korpics, M.; Rodriguez, K.; Colón-Meléndez, L.; Kim, S.-H.; Pine, D.; Yi, G.-R. Shaping colloids for self-assembly. Nat. Commun. 2013, 4, 1688.

(9) Meester, V.; Verweij, R. W.; van der Wel, C.; Kraft, D. J. Colloidal recycling: reconfiguration of random aggregates into patchy particles. ACS Nano 2016, 10, 4322-4329.

(10) Stöber, W.; Fink, A.; Bohn, E. Controlled growth of monodisperse silica spheres in the micron size range. J. Colloid Interface Sci. 1968, 26, 62-69.

(11) Goodwin, J. W.; Hearn, J.; Ho, C. C.; Ottewill, R. H. Studies on the preparation and characterisation of monodisperse polystyrene latices, III. Preparation wihout added surface active agents. Colloid Polym. Sci. 1974, 252, 464-471.

(12) Antl, L.; Goodwin, J. W.; Hill, R. D.; Ottewill, R. H.; Owens, S. M.; Papworth, S.; Waters, J. A. The preparation of poly(methyl methacrylate) latices in non-aqueous media. Colloids Surf. 1986, 17, $67-78$.

(13) Liu, Y.; Edmond, K. V.; Curran, A.; Bryant, C.; Peng, B.; Aarts, D. G. A. L.; Sacanna, S.; Dullens, R. P. A. Core-shell particles for simultaneous 3D imaging and optical tweezing in dense colloidal materials. Adv. Mater. 2016, 28, 8001-8006.

(14) Obey, T. M.; Vincent, B. Novel monodisperse "silicone oil"/ water emulsions. J. Colloid Interface Sci. 1994, 163, 454-463.

(15) Sacanna, S.; Kegel, W. K.; Philipse, A. P. Thermodynamically stable pickering emulsions. Phys. Rev. Lett. 2007, 98, 158301.

(16) Kraft, D. J.; De Folter, J. W. J.; Luigjes, B.; Castillo, S. I. R.; Sacanna, S.; Philipse, A. P.; Kegel, W. K. Conditions for equilibrium solid-stabilized emulsions. J. Phys. Chem. B 2010, 114, 10347.

(17) Sacanna, S.; Kegel, W. K.; Philipse, A. P. Spontaneous oil-inwater emulsification induced by charge-stabilized dispersions of various inorganic colloids. Langmuir 2007, 23, 10486-10492.

(18) Sacanna, S.; Philipse, A. P. A generic single-step synthesis of monodisperse core/shell colloids based on spontaneous Pickering emulsification. Adv. Mater. 2007, 19, 3824-3826.

(19) Miller, J. D.; Hoh, K.-P.; Ishida, H. Studies of the simulation of silane coupling agent structures on particulate fillers; the $\mathrm{pH}$ effect. Polym. Compos. 1984, 5, 18-28.

(20) Arkles, B.; Steinmetz, J. R.; Zazyczny, J.; Mehta, P. Factors contributing to the stability of alkoxysilanes in aqueous solution. $J$. Adhes. Sci. Technol. 1992, 6, 193-206.

(21) Piana, K.; Schubert, U. Catalyst Influence on the molar mass distribution of hydrolyzed (3-glycidoxypropyl)trimethoxysilane and (3-methacryloxypropyl)trimethoxysilane. Chem. Mater. 1994, 6, 15041508.

(22) Altmann, S.; Pfeiffer, J. The hydrolysis/condensation behaviour of methacryloyloxyalkylfunctional alkoxysilanes: structure-reactivity relations. Monatsh. Chem. 2003, 134, 1081-1092.

(23) Brochier-Salon, M.-C.; Bayle, P.-A.; Abdelmouleh, M.; Boufi, S.; Belgacem, M. N. Kinetics of hydrolysis and self condensation reactions of silanes by NMR spectroscopy. Colloids Surf., A 2008, 312, 83-91.

(24) Sacanna, S.; Rossi, L.; Philipse, A. P. Oil-in-water emulsification induced by ellipsoidal hematite colloids: evidence for hydrolysismediated self-assembly. Langmuir 2007, 23, 9974-9982.

(25) Šefćík, J.; McCormick, A. V. Thermochemistry of aqueous silicate solution precursors to ceramics. AIChE J. 1997, 43, 27732784.

(26) Šefč́ik, J.; Rankin, S. E.; Kirchner, S. J.; McCormick, A. V. Esterification, condensation, and deprotonation equilibria of trimethylsilanol. J. Non-Cryst. Solids 1999, 258, 187-197.

(27) Morin, C. J.; Geulin, L.; Desbène, A.; Desbène, P. L. Study of the acid hydrolysis of (3-methacryloxypropyl)trimethoxysilane by capillary electrophoresis-ion-trap mass spectrometry. J. Chromatogr. A 2004, 1032, 327-334.

(28) Kraft, D. J.; Luigjes, B.; de Folter, J. W. J.; Philipse, A. P.; Kegel, W. K. Evolution of equilibrium Pickering emulsions - a matter of time scales. J. Phys. Chem. B 2010, 114, 12257.

(29) Sacanna, S.; Irvine, W. T. M.; Rossi, L.; Pine, D. J. Lock and key colloids through polymerization-induced buckling of monodisperse silicon oil droplets. Soft Matter 2011, 7, 1631-1634.

(30) van Blaaderen, A.; Vrij, A. Synthesis and characterization of colloidal dispersions of fluorescent, monodisperse silica spheres. Langmuir 1992, 8, 2921-2931.

(31) Verhaegh, N. A. M.; van Blaaderen, A. Dispersions of rhodamine-labeled silica spheres: synthesis, characterization, and fluorescence confocal scanning laser microscopy. Langmuir 1994, 10, $1427-1438$

(32) Verweij, R. W.; van der Wel, C. semtracking: determining the size distribution of particles in SEM images. Zenodo 2016, 10.5281/ zenodo. 45575 .

(33) Zwanikken, J.; Ioannidou, K.; Kraft, D.; van Roij, R. Reversible emulsification controlled by ionic surfactants and responsive nanoparticles. Soft Matter 2011, 7, 11093. 